\title{
Restrictions for different functional forms of the matching function
}

\author{
Ausias Ribó ${ }^{1,2} \cdot$ Montserrat Vilalta-Bufi ${ }^{1,2,3}$
}

Received: 25 March 2019 / Accepted: 10 September 2019 / Published online: 19 September 2019

(c) The Author(s) 2019

\begin{abstract}
We provide bounds on the parameters of matching functions such that the job-finding rate and the vacancy-filling rate are below 1 . We do that in the context of the canonical search and matching model with a Pissarides-type free-entry condition. We find that the restrictions for a Cobb-Douglas matching function with increasing returns to scale are rather restrictive, involving an upper bound to future expected profits and the number of job searchers. In contrast, for functional forms with constant returns to scale (Cobb-Douglas, CES) the restrictions involve only parameters or an upper bound to the future expected profits. The paper also investigates when a job-finding rate (vacancy filling rate) below one can restrict the vacancy filling rate (job-finding rate) to be below and strictly bounded away from one. We provide the bounds implied by these "interior equilibria."
\end{abstract}

Keywords Matching function · Random matching · Interior equilibrium

\section{JEL Classification J6}

\section{Introduction}

The use of matching functions in economic models allows for the introduction of market frictions in a tractable fashion. The matching function describes how the number of job searchers and the number of open vacancies relate to the number of new job matches that occur within a period. It is common in this literature to interpret the number of matches per job searcher as the average job-finding rate. This class of models has proven important when studying unemployment and its relationship with other

\footnotetext{
$\bowtie \quad$ Montserrat Vilalta-Bufí montsevilalta@ub.edu

1 CREB, Universitat de Barcelona, Barcelona, Spain

2 Department of Economics, Universitat de Barcelona, Barcelona, Spain

3 BEAT, Universitat de Barcelona, Barcelona, Spain
} 
phenomena. The extensive body of literature on matching models shows its many applications (see Petrongolo and Pissarides 2001; Rogerson et al. 2005; Yashiv 2007 for surveys).

In this paper, we find the restrictions that different functional forms of the matching function must satisfy to ensure that the number of matches per job searcher can be interpreted as a job-finding probability. We do so in the context of the Pissarides matching model with a free-entry condition for firms. Our results are relevant in a discrete time framework, when the job-finding rate is a probability and, therefore, must lie below 1 . They also apply to the empirical estimations of the matching function when variables are measured in a discrete time framework (monthly, quarterly, etc). These restrictions are useful in those models where the matching function is simply an instrument to introduce frictions in the labor market without explicitly modeling their micro-foundations (Blanchard and Gali 2010). The role of these frictions is then to allow for the existence of unemployment in equilibrium. In these cases, it is natural to interpret the number of matches per job searcher as a probability, i.e., the probability to find a job by a worker.

The paper is organized as follows. In the next section, we specify the basic framework of our analysis. In Sect. 3, we derive the theoretical restrictions that the most commonly assumed matching functions (a Cobb-Douglas and two CES matching functions) must satisfy to get an interior equilibrium. We also provide the bounds on the probability to find a job and to fill in a vacancy originated as a result of these restrictions. We summarize our results in the concluding section.

\section{Basic framework}

Let us denote by $M$ the number of new matches created in one period, which is assumed to be a function of the number of job seekers $(S)$ and the number of open vacancies $(V)$ at the beginning of the period, $M=m(S, V) .{ }^{1}$ We assume, as it is common in this literature, that the matching function is increasing in its arguments and concave. In this setup, the average probability of finding a job by a worker is the number of matches per job searcher, $p=m(S, V) / S$, and the average probability of filling in a vacancy by a firm is the number of matches per vacancy, $q=m(S, V) / V$.

We assume free entry in vacancies as in Pissarides (1985), with a positive cost of opening a vacancy. The number of posted vacancies is determined by:

$$
q B=\kappa,
$$

where $q$ is the probability to fill in a vacancy, $B$ are the expected future profits of a filled vacancy for the firm and $\kappa>0$ is the cost of posting a vacancy. Note that $B$ is endogenous in a general model, and it includes any effects of the separation rate on the expected future profits. It is, however, independent of the number of job searchers. This condition applies to a large variety of random matching models, from those with exogenous or endogenous separation rate to those with match-specific productivity.

\footnotetext{
1 Time subscripts have been omitted to enhance readability.
} 
These models will differ in their specification of $B$ (Rogerson et al. 2005). For the purpose of this paper, though, we do not need to specify any particular form.

Definition 1 An interior equilibrium occurs when the number of matches is bounded from above by the number of job searchers and the number of vacancies in the economy $(M<\min \{S, V\})$.

Definition 1 implies that the probability of finding a job $(p)$ and the probability of filling in a vacancy $(q)$ are both below unity. Notice from (1) that the condition $q<1$ is automatically satisfied in equilibrium, since vacancies will be open if and only if $B>\kappa$. Otherwise, the expected benefits of a filled vacancy would be lower than its cost and no one would have incentives to open any vacancy. ${ }^{2}$ This result holds in equilibrium for any functional form of the matching function. However, the matching function will be important in determining whether the probability of finding a job is lower than 1 . In the following section, we study the conditions under which several matching functions lead to an interior equilibrium. Since we proved that $q<1$ in any equilibrium, it is only left to check whether $p<1$ holds in equilibrium.

\section{Theoretical restrictions for an interior equilibrium}

In this section, we derive the theoretical restrictions that must be satisfied for a matching function to be consistent with the existence of an interior equilibrium. We study the restrictions for the Cobb-Douglas and two CES specifications of the matching function. We also provide the bounds on the probability to find a job and to fill in a vacancy originated by these restrictions.

\subsection{Cobb-Douglas matching function}

In this section, we analyze the necessary restrictions for a Cobb-Douglas matching function to have an interior equilibrium. We also distinguish the implications of these restrictions under different returns to scale on the matching technology.

The Cobb-Douglas matching function has been used extensively in the literature. Most of the empirical estimations of the matching function use this specification and usually find constant returns to scale, although some studies find increasing returns (Petrongolo and Pissarides 2001; Yashiv 2007).

Proposition 1 In a general equilibrium random matching model with a Cobb-Douglas matching function, $m=A S^{\alpha} V^{\beta}$, where $A>0$ is a scale parameter, $\alpha \in(0,1)$ and $\beta \in(0,1)$, an interior equilibrium exists if and only if $B>\kappa$ and $S^{\alpha+\beta-1} B^{\beta}<\frac{\kappa^{\beta}}{A}$.

\footnotetext{
2 We follow the common assumption in the literature that each vacancy is open for one individual and the cost of opening the vacancy occurs each time you recruit a new worker.
} 
Proof Let us define the matching function to be Cobb-Douglas, $m=A S^{\alpha} V^{\beta}$, where $A>0, \alpha \in(0,1)$ and $\beta \in(0,1)$. An interior equilibrium exists if $M<S .{ }^{3}$

To prove that $M<S$, we substitute $q$ in (1) for $m / V$, where $m$ has the assumed functional form and solve for $V$. Then, we substitute $V$ in $M<S$, where $M$ is defined by the Cobb-Douglas matching function. Rearranging, we obtain the condition in Proposition 1:

$$
S^{\alpha+\beta-1} B^{\beta}<\frac{\kappa^{\beta}}{A} .
$$

Corollary 1 Different returns to scale in the matching function imply different conditions to have an interior equilibrium:

(a) With constant returns to scale $(\alpha+\beta=1)$, there is an upper bound to the expected future profits of a filled vacancy: $B^{\beta}<\frac{\kappa^{\beta}}{A}$,

(b) With increasing returns to scale $(\alpha+\beta>1)$, there is a joint upper bound to the number of job searchers and the expected future profits of a filled vacancy: $B^{\beta}<\frac{\kappa^{\beta}}{A S^{\alpha+\beta-1}}$,

(c) With decreasing returns to scale $(\alpha+\beta<1)$, there is an interrelated upper bound to the expected future profits of a filled vacancy and a lower bound to the number of job searchers: $B^{\beta}<\frac{\kappa^{\beta}}{A} S^{1-\alpha-\beta}$.

Proposition 1 states the necessary conditions for any Cobb-Douglas matching function to lead to an interior equilibrium. In all cases, there is an upper bound to the expected future profits of a filled vacancy. This is due to the freeentry assumption of vacancies. In this framework, the number of vacancies is a jump variable that adjusts immediately to the equilibrium, while the number of searchers is generally a state variable. ${ }^{4}$ Then, to make sure $S>M$ we have to limit $M$ by restricting the amount of vacancies. We do that by setting an upper bound to the expected profits. As Corollary 1 shows, in the case of increasing returns to scale (IRS), this upper bound decreases with the number of searchers, while in the case of decreasing returns to scale (DRS) it is increasing with the number of searchers. Therefore, given a number of searchers, the constraint is more stringent when there are IRS than when there are DRS. The constraint is independent of the number of searchers in the case of constant returns to scale (CRS).

Notice also that in the case of CRS, a Cobb-Douglas matching function with scale parameter $(A)$ larger or equal to 1 cannot be a good representation of the labor market frictions, since it would imply that any job searcher fills a vacancy with probability one. $^{5}$

\footnotetext{
3 Recall that since we assume $B>\kappa$, the condition $M<V$ is satisfied in any equilibrium.

${ }^{4}$ The number of searchers might not be a pure state variable in models with search intensity, for instance. The results apply for these cases too.

5 Recall that since $B>\kappa$ and $\beta>0,(\kappa / B)^{\beta}<1$.
} 


\subsection{CES matching function}

Another matching function commonly used in the literature takes a CES specification. In this section, we analyze two CES matching functions and the conditions they must satisfy to ensure an interior equilibrium.

Proposition 2 In a general equilibrium random matching model with a matching function represented by a CES function $m=A\left(\eta S^{\sigma}+(1-\eta) V^{\sigma}\right)^{1 / \sigma}$, where $A>0$ is a scale parameter, $\eta \in(0,1), \sigma<1$ and $1 /(1-\sigma)$ is the elasticity of substitution between job searchers and vacancies, an interior equilibrium exists if and only if $B>\kappa$ and:
(a) $\sigma<0, \frac{(1-\eta)^{-1 / \sigma} \kappa}{A}<B<\left(\frac{A^{-\sigma}-\eta}{1-\eta}\right)^{1 / \sigma} \kappa$ or,
(b) $\sigma>0$ and $B<\left(\frac{A^{-\sigma}-\eta}{1-\eta}\right)^{1 / \sigma} \kappa$.

Proof Let us define the matching function to be CES such that

$$
m=A\left(\eta S^{\sigma}+(1-\eta) V^{\sigma}\right)^{\frac{1}{\sigma}},
$$

where $A>0, \eta \in(0,1)$ and $\sigma<1$. To prove that $M<S$, we substitute $q$ in (1) for $m / V$, where $m$ has its assumed functional form and derive the following equation:

$$
\eta\left(\frac{S}{V}\right)^{\sigma}=\left(\frac{\kappa}{A B}\right)^{\sigma}-(1-\eta) .
$$

Notice that for the market tightness $(V / S)$ to be positive, the RHS of the previous equation must be positive. Therefore, in any equilibrium $(\kappa / A B)^{\sigma}>(1-\eta)$. This translates into $B<(1-\eta)^{-1 / \sigma}(\kappa / A)$ if $\sigma>0$ and $B>(1-\eta)^{-1 / \sigma}(\kappa / A)$ if $\sigma<0$.

Using Eq. (2), we solve for $V$ and use this expression to substitute $V$ in the condition $S>M$, where $M$ is defined by the CES matching function above. We obtain the following inequality:

$$
\left(\left(\frac{\kappa}{A B}\right)^{\sigma}-(1-\eta)\right)^{1 / \sigma}>\frac{\kappa}{B} \eta^{1 / \sigma}
$$

This inequality is satisfied if and only if $\frac{B}{\kappa}<\left(\frac{A^{-\sigma}-\eta}{1-\eta}\right)^{1 / \sigma}$ for any $\sigma<0$ or $\frac{B}{\kappa}<\left(\frac{A^{-\sigma}-\eta}{1-\eta}\right)^{1 / \sigma}$ for any $\sigma>0$. Putting all the conditions together, we find the result in Proposition 2. Notice that $\frac{(1-\eta)^{-1 / \sigma}}{A}<\left(\frac{A^{-\sigma}-\eta}{1-\eta}\right)^{1 / \sigma}$ is always satisfied as long as $A^{\sigma} \eta>0$, which is true by assumption.

Proposition 3 In a general equilibrium random matching model with a matching function represented by the following CES function, $m=\left(S^{\sigma}+V^{\sigma}\right)^{1 / \sigma}$, where $\sigma<1$ and $1 /(1-\sigma)$ is the elasticity of substitution between job searchers and vacancies; the equilibrium is interior if and only if $B>\kappa$ and $\sigma<0$. 
Proof Let us define the matching function to be CES such that

$$
m=\left(S^{\sigma}+V^{\sigma}\right)^{\frac{1}{\sigma}}
$$

where $\sigma<1$. In order to prove that $M<S$, we substitute $q$ in (1) for $m / V$, where $m$ has its assumed functional form and solve for $V$. Rearranging, we obtain the following:

$$
\left(\frac{\kappa}{B}\right)^{\sigma}-1=\left(\frac{S}{V}\right)^{\sigma} .
$$

Notice that, given $B>\kappa$, the market tightness $(V / S)$ is positive if and only if $\sigma<0$. Therefore, we need $\sigma<0$ in any equilibrium.

To continue the proof, we solve for $V$ and substitute it in the condition $S>M$, where $M$ is defined as the CES matching function above. We obtain the following inequality:

$$
1>\frac{\kappa}{B} \frac{1}{\left((\kappa / B)^{\sigma}-1\right)^{1 / \sigma}},
$$

which, given $B>\kappa$, is satisfied for any $\sigma<0$.

Propositions 2 and 3 report the necessary conditions for two different CES matching functions to lead to an interior equilibrium. The CES function specified in Proposition 2 has an upper bound on the expected future profits of a filled vacancy, as in the CobbDouglas case. The intuition is the same as before. The upper bound restricts the amount of vacancies in equilibrium, which keeps the number of matches below the number of job searchers. Moreover, since $B>\kappa$, it can be shown that the scale parameter $A$ cannot be larger than 1 .

Additionally, we obtain a lower bound on the expected future profits of a filled vacancy in the case of $\sigma<0$. This lower bound ensures that the interior equilibrium with $q<1$ is compatible with $V>0$. In the case of $\sigma<0$, there is strong complementarity between vacancies and job searchers. The intuition is simple if one considers the extreme case of perfect complementarity. Then, the number of matches would be the determined by the number of vacancies when there are fewer vacancies than job searchers. Therefore, with too few vacancies open, all vacancies would get filled and there would not be an interior equilibrium.

In contrast, the only requirement to get an interior equilibrium with the CES matching function studied in Proposition 3 is to have an elasticity of substitution between job searchers and vacancies below unity, that is, smaller than in the Cobb-Douglas case. Under this matching function, for any degree of complementarity between job searchers and vacancies, the constraints on the expected profits that we found in Proposition 2 are always satisfied.

From the empirical side, some papers estimate a CES matching function such as Blanchard and Diamond (1989) and Shimer (2005), although they cannot reject that $\sigma=0$, that is, a Cobb-Douglas specification. We could not find any paper where the matching function has a CES specification with $\sigma>0$. In general, empirical studies 
mostly support a Cobb-Douglas matching function. From the theoretical side, a microfoundation for the general CES matching function is provided in Stevens (2007), while the latter CES specification can represent the aggregate matching function in models with micro-markets that suffer disequilibrium (Petrongolo and Pissarides 2001, p. 407). The latter specification is also equivalent to the one used by den Haan et al. (2000). They study the propagation of aggregate shocks in a dynamic general equilibrium with labor frictions represented by the following matching function, where $\rho=-\sigma$ :

$$
m(S, V)=\frac{S V}{\left(S^{\rho}+V^{\rho}\right)^{1 / \rho}}
$$

They recognize that with a Cobb-Douglas matching function truncation is necessary to rule out matching probabilities greater than unity (den Haan et al. 2000, p. 485). In this section, we showed under which conditions this is true. In this section, we demonstrate that the matching function they propose leads to an interior equilibrium as long as $\rho>0(\sigma<0)$. Although den Haan et al. (2000) do not explicitly state this condition in their paper, their calibration is consistent with our results.

\subsection{Probabilities consistent with an interior equilibrium}

In this subsection, we provide the inverse mapping analysis. We introduce the constraints found in the previous sections into the probability to find a job $(p)$ and the probability to fill in a vacancy $(q)$. This allows us to study whether they are bounded in any interior equilibrium.

Proposition 4 In an interior equilibrium of a random matching model with free-entry condition, the probability to find a job $(p)$ and the probability to fill in a vacancy $(q)$ satisfy the following conditions:

- If the matching function is Cobb-Douglas,

$p \in\left(A^{\frac{1}{1-\beta}} S^{\frac{\alpha+\beta-1}{1-\beta}}, 1\right)$ and $q \in\left(A^{\frac{1}{\beta}} S^{\frac{\alpha+\beta-1}{\beta}}, 1\right)$. These ranges are non-empty as long as $A<S^{1-\alpha-\beta}$.

- If the matching function is CES with $\sigma<0$, $p \in(0,1)$ and $q \in\left(\left(\frac{1-\eta}{A^{-\sigma}-\eta}\right)^{1 / \sigma}, \min \left\{A(1-\eta)^{1 / \sigma}, 1\right\}\right)$.

- If the matching function is CES with $\sigma>0$,

$$
p \in\left(\left(\frac{\eta}{A^{-\sigma}-(1-\eta)}\right)^{1 / \sigma}, 1\right) \text { and } q \in\left(\left(\frac{1-\eta}{A^{-\sigma}-\eta}\right)^{1 / \sigma}, 1\right) \text {. }
$$

- When the matching function is $m=\left(S^{\sigma}+V^{\sigma}\right)^{1 / \sigma}$, then $p \in(0,1)$ and $q \in(0,1)$.

Proof Let us first find the probability range of $p$ for the Cobb-Douglas case. We substitute $q$ in (1) for $m / V$, where $m$ has the assumed functional form and we solve for $V$. Then, we substitute $V$ in $p=A S^{\alpha} V^{\beta-1}$. This gives us: 


$$
p=A^{\frac{1}{1-\beta}} S^{\alpha-1+\frac{\alpha \beta}{1-\beta}}\left(\frac{B}{\kappa}\right)^{\frac{\beta}{1-\beta}}
$$

Then, using the fact that $1<\frac{B}{\kappa}<\left(\frac{S^{1-\alpha-\beta}}{A}\right)^{1 / \beta}$, we obtain the range of $p$. The range is non-empty if $A<S^{1-\alpha-\beta}$.

When the matching function is CES, $m=A\left(\eta S^{\sigma}+(1-\eta) V^{\sigma}\right)^{1 / \sigma}$, we obtain $p=A\left[\eta+(1-\eta)\left(\frac{V}{S}\right)^{\sigma}\right]^{1 / \sigma}$. From the free-entry condition, we get an expression for $V / S$ that depends on $B / \kappa$, which we can substitute in $p$. Then, we must distinguish between two cases: positive and negative $\sigma$. From Proposition 2, we know that in an interior equilibrium $\frac{(1-\eta)^{-1 / \sigma}}{A}<\frac{B}{\kappa}<\left(\frac{A^{-\sigma}-\eta}{1-\eta}\right)^{1 / \sigma}$ when $\sigma<0$. Then, it follows that $p \in(0,1)$.

When $\sigma>0$, then the constraint to be satisfied in any interior equilibrium is $1<\frac{B}{\kappa}<\left(\frac{A^{-\sigma}-\eta}{1-\eta}\right)^{1 / \sigma}$. This leads to $p \in\left(\left(\frac{\eta}{A^{-\sigma}-(1-\eta)}\right)^{1 / \sigma}, 1\right)$, where the lower bound is positive since $A<1$.

For all cases, from the free-entry condition we directly obtain that $q=k / B$, which given the constraints in $B / \kappa$, gives us the range of $q$. Notice that for the CES with $\sigma<0$, the lower bound of $q$ is always positive because $A<1$ in an interior equilibrium, while the upper bound could be below or above 1 . In any case, it can be proven that the upper bound is always larger than the lower bound. For the case CES with $\sigma>0$, the ranges are non-empty given that $A<1$.

We observe that in all cases except for the latter CES function, the range of the probability to fill in a vacancy and find a job is smaller than the $(0,1)$ interval.

\section{Conclusions}

This paper studies the theoretical properties that different functional forms of matching functions must satisfy to have an interior equilibrium in a general equilibrium random matching model with free-entry condition for vacancies. We establish and compare the restrictions needed to make sure that the usual interpretation of the ratio of matches to unemployed as a job-finding probability is consistent with the assumption of interior equilibria. We find that the restrictions for a CobbDouglas function with increasing returns to scale are rather restrictive, involving an upper bound to future expected profits and the number of job searchers. In contrast, for functional forms with constant returns to scale (Cobb-Douglas, CES) the restrictions involve only parameters or an upper bound to the future expected profits. We also show the range that the job-finding probability and the probability to fill in a vacancy can take in an interior equilibrium. Our results provide theoretical results to help choose among different functional forms of a matching function.

Acknowledgements The authors wish to thank an anonymous referee, Thijs van Rens, José Ignacio Silva, Xavier Raurich, Lorenzo Burlon, Aleksander Kucel and seminar participants at University of Barcelona for helpful comments and suggestions. 


\section{Compliance with ethical standards}

Conflict of interest The authors declare that they have no conflict of interest.

Ethical approval This article does not contain any studies with human participants or animals performed by any of the authors.

Informed consent Both authors of this paper agree with the submission of the paper to the Journal of the Spanish Economic Association (SERIEs). The authors declare no ethical issues or issues needing informed consent.

Open Access This article is distributed under the terms of the Creative Commons Attribution 4.0 International License (http://creativecommons.org/licenses/by/4.0/), which permits unrestricted use, distribution, and reproduction in any medium, provided you give appropriate credit to the original author(s) and the source, provide a link to the Creative Commons license, and indicate if changes were made.

\section{References}

Blanchard OJ, Diamond P (1989) The Beveridge curve. Brook Pap Econ Act 20(1):1-76

Blanchard OJ, Galí J (2010) Labor markets and monetary policy: a New Keynesian model with unemployment. Am Econ J Macroecon 2:1-30

den Haan WJ, Ramey G, Watson J (2000) Job destruction and propagation of shocks. Am Econ Rev 90(3):482-498

Petrongolo B, Pissarides CA (2001) Looking into the black box: a survey of the matching function. J Econ Lit 39(2):390-431

Pissarides CA (1985) Short-run equilibrium dynamics of unemployment, vacancies and real wages. Am Econ Rev 75:676-690

Rogerson R, Shimer R, Wright R (2005) Search-theoretic models of the labor market: a survey. J Econ Lit 43(4):959-988

Shimer R (2005) The cyclical behavior of equilibrium unemployment and vacancies. Am Econ Rev 95(1):25-49

Stevens M (2007) New microfoundations for the aggregate matching function. Int Econ Rev 48(3):847-868

Yashiv E (2007) Labor search and matching in macroeconomics. Eur Econ Rev 51(8):1859-1895

Publisher's Note Springer Nature remains neutral with regard to jurisdictional claims in published maps and institutional affiliations. 